\title{
Elevated Serum Potassium Concentration Alleviates Cerebral Ischemia-Reperfusion Injury via Mitochondrial Preservation
}

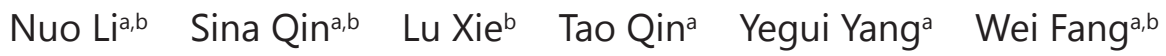 \\ Meng-hua Chen ${ }^{\mathrm{a}}$ \\ Intensive Care Unit, The Second Affiliated Hospital of Guangxi Medical University, Nanning, Guangxi, \\ ${ }^{b}$ Guangxi Medical University, Nanning, Guangxi, China
}

\section{Key Words}

$\mathrm{Kcl} \cdot$ Ischemia-reperfusion injury $\bullet$ Mitochondria $•$ Apoptosis

\begin{abstract}
Background/Aims: The anti-apoptotic effect of an increase in the extracellular concentration of potassium ([K+]) has been confirmed in vitro. However, it is not yet known whether elevated serum $\left[\mathrm{K}^{+}\right]$exerts a cerebroprotective effect in vivo. In this study, we aimed to explore the effect of elevated serum $\left[\mathrm{K}^{+}\right]$in a rat model of middle cerebral artery occlusion and reperfusion (MCAO/R). Methods: Rats subjected to 90 -min $\mathrm{MCAO}$ received $2.5 \% \mathrm{KCL}, 1.25 \%$ $\mathrm{KCL}$, or a normal saline solution at a dose of $3.2 \mathrm{~mL} / \mathrm{kg}$ at the onset of reperfusion. Rats that were subjected to vascular exposure and ligation without MCAO were defined as the Sham group. Serum $\left[\mathrm{K}^{+}\right]$was determined using a blood gas analyzer at $1 \mathrm{~min}$ after medicine administration. At $24 \mathrm{~h}$ post-reperfusion, rat brains were harvested and processed for $2 \%$ 2,3,5-triphenyltetrazolium chloride staining, terminal deoxynucleotidyl transferase-mediated 2 '-deoxyuridine 5'-triphosphate-biotin nick end labeling staining, detection of caspase-3 and cleaved-caspase- 3 by western blotting, detection of reactive oxygen species (ROS) by dihydroethidium staining, and observation of mitochondrial structure by electron microscopy. In addition, malondialdehyde (MDA), adenosine triphosphate (ATP), total superoxide dismutase (T-SOD), cytochrome $C$ oxidase (COX) activity, and mitochondrial permeability transition pore (MPTP) opening were measured using detection kits. Results: The results showed that elevated serum $\left[\mathrm{K}^{+}\right]$decreased cerebral injury and apoptosis, reduced ROS and MDA levels and MPTP opening, increased ATP levels and cytochrome $C$ oxidase activity, and improved mitochondrial ultrastructural changes, although there was no significant difference in T-SOD activity. Conclusion: These findings suggested that elevated serum $\left[\mathrm{K}^{+}\right]$could alleviate cerebral ischemia-reperfusion injury and the mechanism may be associated with the preservation of mitochondrial function.




\section{Cellular Physiology Cell Physiol Biochem 2018;48:1664-1674 \begin{tabular}{l|l} 
and Biochemistry Published 10.1159/000492289 2,2018 & $\begin{array}{l}\text { (c) } 2018 \text { The Author(s). Published by S. Karger AG, Basel } \\
\text { www.karger.com/cpb }\end{array}$ \\
\hline
\end{tabular}}

Li et al.: Cerebral Protection by Serum Potassium Regulation

\section{Introduction}

When cerebral ischemia occurs, it is crucial to restore blood supply as soon as possible to avoid neuronal loss in the ischemic area. However, the restoration of blood supply may result in ischemia-reperfusion injury (IRI), due at least in part to the excessive generation of reactive oxygen species (ROS) [1-4]. ROS are formed in the mitochondria as a natural byproduct of metabolism, and they have important roles in the signaling pathways for cellular proliferation, survival, and differentiation under normal physiological conditions [5]. However, under pathophysiological conditions, some pathological factors, such as IRI, can cause mitochondrial dysfunction, which can, consequently, lead to a dramatic increase in the levels of ROS without a corresponding increase in the ability to eliminate these species. ROS can degrade polyunsaturated lipids to damage cells [6, 7]. Therefore, it may be a therapeutic option to protect mitochondrial function by maintaining the balance between ROS generation and elimination to alleviate IRI.

On the other hand, cell apoptosis and shrinkage can be induced by a deficiency or efflux of intracellular potassium $[8,9]$. In addition, an increase in the extracellular concentration of potassium $\left(\left[\mathrm{K}^{+}\right]\right)$or inhibition of potassium efflux can improve mitochondrial function and exert an anti-apoptotic effect on cells $[10,11]$. In animal experiments, beneficial effects also seem to be produced when serum $\left[\mathrm{K}^{+}\right]$is increased. For example, rats in a cardiac arrest (CA) model established by $\mathrm{KCl}$ injection were easier to resuscitate than those in one established by electrical stimulation. Furthermore, CA rats induced by $\mathrm{KCl}$ injection could tolerate a longer period of ischemia and showed less neural injury than those induced by electrical stimulation [12-14]. Since the key biochemical difference between these two CA rat models is serum $\left[\mathrm{K}^{+}\right]$, it is rational to hypothesize that elevating serum $\left[\mathrm{K}^{+}\right]$by the venous administration of a $\mathrm{KCl}$ solution could provide some protection to the brain against IRI. Therefore, in the present study, we examined whether elevated serum $\left[\mathrm{K}^{+}\right]$could alleviate cerebral IRI using a rat model of focal cerebral ischemia with middle cerebral artery occlusion and reperfusion (MCAO/R). In addition, the underlying mechanism was studied.

\section{Materials and Methods}

\section{Animal preparation and the focal cerebral MCAO/R model}

Healthy adult male Sprague-Dawley rats (body weight 250-280 g, age 8 weeks) were provided by the Experimental Animal Center of Guangxi Medical University. All rats were handled according to the Guidelines for the Care and Use of Laboratory Animals of the National Institutes of Health (NIH) and the experimental protocol was approved by the Animal Experiment Committee of Guangxi Medical University.

The rats were fasted for $12 \mathrm{~h}$ with access to water ad libitum before surgery. The rats were anesthetized with an intraperitoneal injection of $\%$ pentobarbital sodium $(30 \mathrm{mg} / \mathrm{kg}$ ). According to the MCAO procedure described previously [15], the right common carotid artery (CCA), internal carotid artery (ICA), and external carotid artery (ECA) were exposed by a midline incision on the neck. A monofilament nylon suture (L3600; Guangzhou Jia Ling Biological Technology Co., Ltd, Guangdong, China) with an external diameter of $0.28 \mathrm{~mm}$ and a silicone-coated tip was inserted from the bifurcation through the ECA into the ICA to a distance of approximately $18-20 \mathrm{~mm}$. After $90 \mathrm{~min}$ of MCAO, the suture was withdrawn, blood flow was restored, and the ECA was ligated. During surgery, the rectal temperature of the rats was maintained at $37.0 \pm 0.5^{\circ} \mathrm{C}$ with a heating lamp. The rats in the Sham group underwent exposure of the right CCA, ICA, and ECA followed by ECA ligation without MCAO. All surgical procedures were completed by a skilled investigator.

At the onset of reperfusion, the rats were assigned randomly to receive a $1.25 \% \mathrm{KCl}$ solution (lowdose group, LD group), $2.5 \% \mathrm{KCl}$ solution (high-dose group, HD group), or $0.9 \%$ normal saline solution (NS group) at a dosage of $3.2 \mathrm{~mL} / \mathrm{kg}$. Administration was performed through the right jugular vein with a micro pump at an infusion rate of $8 \mathrm{~mL} / \mathrm{h}$. Administration of the test solutions was completed by another skilled investigator who followed the instructions provided in a sealed envelope that were prepared by a third investigator. Rats that experienced an anesthetic or surgical accident were excluded. The criteria that were used to determine whether the rats would undergo further experiments are detailed in the following 


\section{Cellular Physiology Cell Physiol Biochem 2018;48:1664-1674

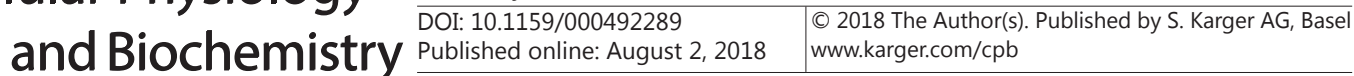

Li et al.: Cerebral Protection by Serum Potassium Regulation

paragraphs. At 1 min after administration, serum $\left[\mathrm{K}^{+}\right]$of the rats was determined using an arterial blood gas analyzer ( $\mathrm{n}=8$ /group). The rats were then sutured and placed singly in a cage with dry bedding and housed in an air-conditioned and quiet room (room temperature $26^{\circ} \mathrm{C}$ ). The rats had free access to water and food.

\section{Evaluation of brain infarct volume}

To evaluate brain infarct volume, the rats ( $n=8$ /group) were anesthetized deeply with an intraperitoneal injection of $2 \%$ pentobarbital sodium $(30 \mathrm{mg} / \mathrm{kg})$ at $24 \mathrm{~h}$ after reperfusion. Then, the brains were removed quickly, ensuring that they had no subarachnoid hemorrhage, the cerebellum and frontal pole in the suprachiasmatic nucleus were removed, and the rest of the brain was sliced into 2-mm-thick coronal sections (a total of 5 total on average) for staining. Staining was performed in the dark using a 2\% 2, 3,5-triphenyltetrazolium chloride (TTC) (Sigma-Aldrich, St. Louis, MO, USA) solution for $30 \mathrm{~min}$ in a water bath at $37{ }^{\circ} \mathrm{C}$, and the samples were incubated in $4 \%$ paraformaldehyde in phosphate-buffered saline (pH 7.4) for $12 \mathrm{~h}$. Infarcted tissue was stained white, while normal tissue was stained red. Infarct volume in each group after TTC staining was assessed by a researcher blinded to group assignment. The section area was evaluated using Image J software (v1.33; NIH, Bethesda, MD, USA), and color quantification was determined as follows: percentage of infarction $=$ (measured infarct area - [ipsilateral hemisphere area - contralateral hemisphere area]) / ([ipsilateral hemisphere area + contralateral hemisphere area] [ipsilateral hemisphere area - contralateral hemisphere area]) [16].

\section{Assessment of neuronal apoptosis}

To evaluate neuronal apoptosis, the rats ( $n=8$ /group) were anesthetized with an intraperitoneal injection of $2 \%$ pentobarbital sodium $(30 \mathrm{mg} / \mathrm{kg})$ at $24 \mathrm{~h}$ after reperfusion. The brains were then removed quickly, frozen, and cut into 5- $\mu$ m-thick sections using a CM1950 Cryostat Microtome (Leica, Nussloch, Germany), and the sections underwent terminal deoxynucleotidyl transferase-mediated 2'-deoxyuridine $5^{\prime}$-triphosphate-biotin nick end labeling (TUNEL) staining with an In Situ Cell Death Detection Kit (Roche, Indianapolis, IN, USA), which was used to assess DNA damage as a marker of apoptosis. 4',6-Diamidino-2phenylindole (DAPI; Sigma-Aldrich) was used as a chromogen. TUNEL-positive apoptotic cells exhibited green fluorescence. All cells, including apoptotic cells and non-apoptotic cells, can be stained by DAPI and present with blue fluorescence. The number of green and blue fluorescent cells was counted in the infarcted cortex at 400× magnification using an Olympus BX53 reflected light fluorescence microscope (Olympus, Tokyo, Japan) in five randomly-selected fields. The apoptotic index (AI) was calculated as the number of green fluorescent cells/number of blue fluorescent cells.

\section{Western blot assessment of caspase-3 and cleaved-caspase-3}

Caspase- 3 and cleaved-caspase- 3 were assessed by western blotting. Right ischemic cortices were collected, weighed, and homogenized in a 1:10 (w/v) radio immunoprecipitation assay lysis buffer (P0013B; Beyotime Biotechnology, Shanghai, China) including a $1 \mathrm{mmol} / \mathrm{L}$ phenylmethanesulfonyl fluoride (P0100; Solarbio, Beijing, China) solution using a glass homogenizer. After $30 \mathrm{~min}$, the soluble proteins were collected and centrifuged at $14,000 \times g$ for $15 \mathrm{~min}$ at $4{ }^{\circ} \mathrm{C}$. After centrifugation, total protein concentration was determined using a BCA Protein Assay Reagent Kit (P0010; Beyotime Biotechnology) with the proteins extracted from the supernatant, which were then denatured at $100^{\circ} \mathrm{C}$ for $15 \mathrm{~min}$ in $4 \times$ sodium dodecyl sulfatepolyacrylamide gel electrophoresis (SDS-PAGE) loading buffer (P1015; Solarbio). Samples containing $25 \mu \mathrm{g}$ total protein were separated by $15 \%$ SDS-PAGE and transferred to an Immobilon-P membrane $(0.22 \mu \mathrm{m}$ bore diameter; Millipore, Bedford, MA, USA), which was then blocked by $5 \%$ nonfat milk. Immunoblotting analysis was performed using an anti-caspase-3/cleaved-caspase-3 monoclonal antibody (\#9662, 1:800; Cell Signaling Technology, Danvers, MA, USA). $\beta$-Actin was used to control for equal sample loading in each subcellular fraction (\#8457, 1:1000; Cell Signaling Technology). After 3 washes for 5 min with Tris-buffered saline containing $0.1 \%$ Tween 20 (TBST), the membranes were further incubated with a secondary antibody (\#5151, 1:15000; Cell Signaling Technology) for $2 \mathrm{~h}$ followed by 3 washes with TBST. The optical densities of the protein bands were analyzed by Odyssey (LI-COR Biosciences, Lincoln, NE, USA). Blot bands were quantified with Image J software (v1.33; NIH). The quantified values were expressed as a percentage of $\beta$-actin ( $n=8$ /group). All data were obtained from 3 independent experiments.

\section{KARGER}




\section{Cellular Physiology Cell Physiol Biochem 2018;48:1664-1674 \begin{tabular}{ll|l} 
and Biochemistry Published online: August 2, 2018 & $\begin{array}{l}\text { (C) } 2018 \text { The Author(s). Published by S. Karger AG, Basel } \\
\text { www.karger.com/cpb }\end{array}$
\end{tabular}}

\section{ROS measurement}

Dihydroethidium (DHE; Sigma-Aldrich) was used as an ROS-specific fluorescent probe; DHE and intracellular ROS form ethyl oxide species that can mix with chromosomal DNA to produce red fluorescence, the measurement of which provides an estimate of cellular ROS content. At $24 \mathrm{~h}$ after reperfusion, the rats ( $\mathrm{n}=8$ /group) were anesthetized with an intraperitoneal injection of $2 \%$ pentobarbital sodium (30 mg/ $\mathrm{kg})$. Then, the brains were removed quickly, frozen, and cut into $5-\mu$ m-thick sections using a CM1950 Cryostat Microtome (Leica, Nussloch, Germany), fixed by acetone, and then incubated with $1 \mu \mathrm{mol} / \mathrm{L}$ DHE for 30 min at $37{ }^{\circ} \mathrm{C}$. After incubation, fluorescence was observed using a confocal laser microscope (A1; Nikon, Tokyo, Japan) at an excitation wavelength of $488 \mathrm{~nm}$, and emission was recorded at $543 \mathrm{~nm}$. Images were captured by the confocal laser microscope and were set at $1024 \times 1024$ pixels using a $\times 20$ objective lens. The fluorescence intensity of ethidium-positive cells was quantified by NIS-Elements AR3.2 64-bit image software.

Biochemical analysis: brain $K^{+}, A T P$, and malondialdehyde (MDA) levels and total superoxide dismutase (T-SOD) activity

To determine brain $\mathrm{K}^{+}$, ATP, and MDA levels and T-SOD activity, right ischemic cortices were collected at $24 \mathrm{~h}$ after reperfusion and homogenized. Brain tissue homogenates were centrifuged at $1000 \times g$ at $4{ }^{\circ} \mathrm{C}$ for $15 \mathrm{~min}$. The supernatants were then used for the measurement of $\mathrm{K}^{+}$, ATP, and MDA levels and T-SOD activity with a Potassium Assay Kit, ATP Assay Kit, MDA Assay Kit (TBA method), and T-SOD Assay Kit (hydroxylamine method), respectively (Nanjing Jian Cheng Technology Co, Ltd., Nanjing, China). Tissue protein was quantified using the Coomassie brilliant blue reagent (Nanjing Jian Cheng Technology Co, Ltd.), and ATP and MDA levels and total SOD activity were calculated relative to protein concentration $(\mathrm{n}=8 /$ group).

\section{Electron microscopy}

The rats ( $n=4$ /group) were anesthetized with an intraperitoneal injection of $2 \%$ pentobarbital sodium $(30 \mathrm{mg} / \mathrm{kg})$ at $24 \mathrm{~h}$ after reperfusion, transcardially perfused with saline and $4 \%$ paraformaldehyde, and fixed with $2.5 \%$ glutaraldehyde. The brains were removed quickly and $1 \mathrm{~mm} \times 1 \mathrm{~mm} \times 1 \mathrm{~mm}$ tissues were cut from the right ischemic cortices. Then, the tissues were fixed in $2.5 \%$ glutaraldehyde, dehydrated, embedded, and sectioned at $80 \mathrm{~nm}$ using a Leica EM UC7 ultra microtome. The ultrathin sections were stained with uranium-citric acid and examined using an electron microscope (H-7650; Hitachi, Tokyo, Japan).

\section{Measurement of mitochondrial permeability}

Mitochondria were extracted from the right ischemic cortices using a Tissue Mitochondria Isolation Kit (GENMED Scientifics, Inc., Shanghai, China) according to the manufacturer's instructions. Determination of mitochondrial permeability transition pore (MPTP) opening was performed with an MPTP Fluorescence Detection Kit (GENMED Scientifics, Inc.). Calcein-AM is a dye that selectively aggregates inside mitochondria and produces green fluorescence. The dye is released from the mitochondria when the MPTP opens, and changes in mitochondrial fluorescence reflect the degree of MPTP opening. The fluorescence intensity of mitochondria was determined using a microplate reader (Synergy H1; BioTek, Winooski, VT, USA) at an excitation wavelength of $488 \mathrm{~nm}$ and emission wavelength of $505 \mathrm{~nm}$. Mitochondrial protein was quantified using the Coomassie brilliant blue reagent (GENMED Scientifics, Inc.); mitochondrial fluorescence was calculated relative to protein concentration ( $n=8$ /group).

\section{Determination of cytochrome C oxidase activity}

Cytochrome $\mathrm{C}$ oxidase activity was determined using a Micro Mitochondrial Respiratory Chain Complex IV Activity Assay Kit (BC0945; Solarbio). Mitochondria were extracted from right ischemic cortices according to the guidelines of the kit. Mitochondrial complex IV (cytochrome C oxidase activity) is responsible for the catalytic oxidation of reduced cytochrome $\mathrm{C}$, and finally an electron is transferred to oxygen to produce water. Reduced cytochrome $\mathrm{C}$ at a wavelength $550 \mathrm{~nm}$ has characteristic light absorption features. The decline of the light absorption rate at $550 \mathrm{~nm}$ can reflect mitochondrial complex IV enzyme activity. The light absorption value was determined using a microplate reader (Synergy H1; BioTek, Winooski, VT, USA) at a wavelength of $550 \mathrm{~nm}$. Tissue protein was quantified using the Coomassie brilliant blue reagent (Nanjing 
Jian Cheng Technology Co., Ltd.), and cytochrome C oxidase activity was calculated relative to protein concentration (n $=8$ (group) .

\section{Statistical analysis}

Data analysis was performed using SPSS 22.0 statistical software. Groups were compared with one-way analysis of variance followed by the Student-Newman-Keuls test or Dunn-Bonferroni approach for post hoc comparisons. All data are expressed as the mean \pm standard deviation (SD), and a value of $\mathrm{P}<0.05$ was considered to indicate a statistically significant difference.

\section{Results}

Venous administration of a $\mathrm{KCl}$ solution increases serum $\left[\mathrm{K}^{+}\right]$

The rats were administered a $\mathrm{KCl}$ solution intravenously at the moment of reperfusion after 90 min of occlusion. When the transfusion was finished, serum $\left[\mathrm{K}^{+}\right]$was significantly increased in the LD and HD groups when compared with the NS group ( $\mathrm{P}<0.01$, Fig. 1).

\section{Elevated serum $\left[\mathrm{K}^{+}\right]$reduces infarct volume}

The cerebral infarction volume was evaluated by TTC staining at $24 \mathrm{~h}$ of reperfusion (Fig. 2A). Compared with the NS group, the infarct volume was reduced in the LD group $(P<0.05)$ and HD group $(\mathrm{P}<0.01)$. In addition, the infarct volume of the HD group was markedly decreased compared to the LD group $(\mathrm{P}<0.05)$. The rats in the Sham group did not exhibit any deficits (Fig. 2B).

\section{Elevated serum $\left[\mathrm{K}^{+}\right]$reduces neuronal apoptosis} induced by $I / R$

The effect of elevated serum $\left[\mathrm{K}^{+}\right]$on neuronal apoptosis was evaluated by TUNEL and DAPI staining (Fig. 3A). The NS group showed a significantly increased AI compared with the Sham group. Compared with the NS group, both groups that receiving a KCL solution showed a significantly reduced $\mathrm{AI}(\mathrm{P}<0.01)$. In addition, the AI of the HD group was markedly decreased compared with the LD group $(\mathrm{P}<0.01$, Fig. $3 \mathrm{~B})$.

\section{Elevated serum $\left[K^{+}\right]$reduces the expression of} cleaved-caspase-3

The expression of the apoptosis-related proteins caspase- 3 and cleaved-caspase- 3 was detected by western blot analysis (Fig. 4A). The NS group exhibited the highest expression of cleaved-caspase-3. The expression of cleaved-caspase-3 was decreased in both groups that received a KCL solution compared with the NS group $(\mathrm{P}<0.01)$, although there was no statistically significant difference between both $\mathrm{KCl}$ treatment groups. No statistically

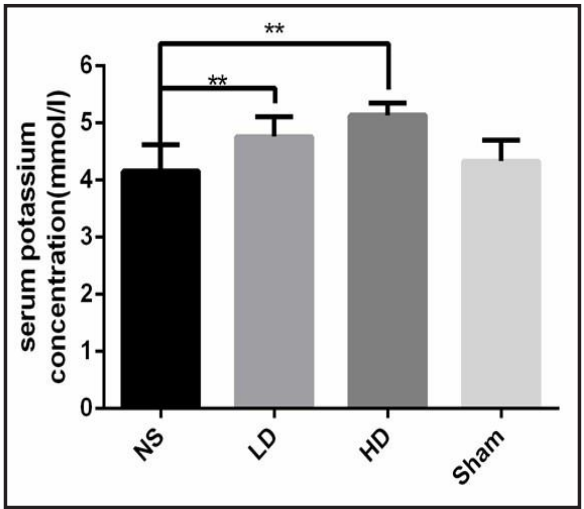

Fig. 1. Venous administration of $\mathrm{KCl}$ solution increases serum $[\mathrm{K}+]$.Data are expressed as the mean $\pm \mathrm{SD}$. ${ }^{* *} \mathrm{P}<0.01$ compared with the NS group ( $\mathrm{n}=8$ /group).

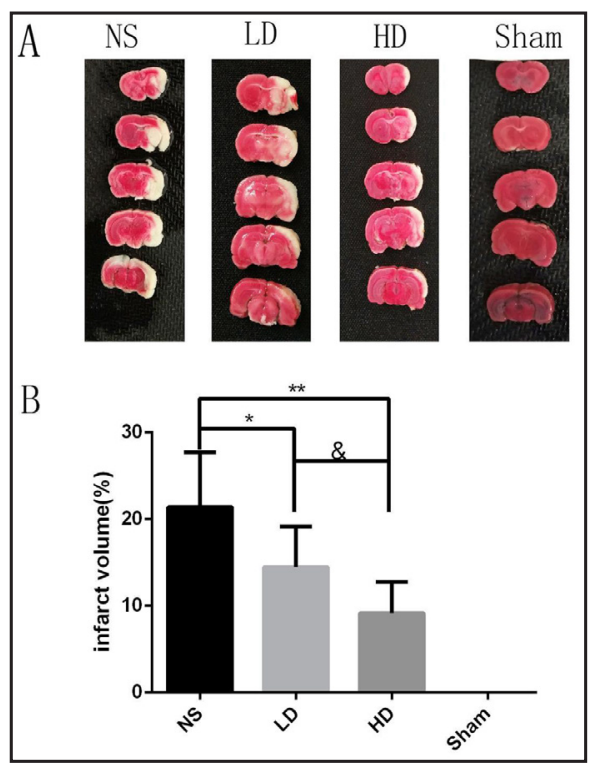

Fig. 2. Elevated serum $[\mathrm{K}+]$ attenuates infarct volume.(A) Representative TTC staining in comparable sections of rat brain in the NS, LD, HD, and Sham groups. (B) Infarct volume measured by TTC staining in each group. Data are expressed as the mean \pm SD. ${ }^{*} \mathrm{P}<0.05$ compared with the NS group; ${ }^{* *} \mathrm{P}<0.01$ compared with the NS group; $\& \mathrm{P}<0.05$ compared with the LD group ( $\mathrm{n}=8 /$ group). 


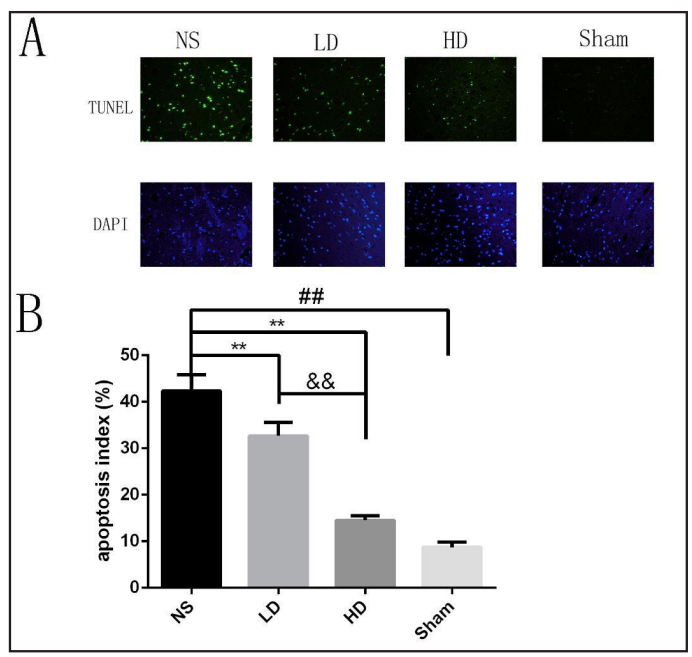

Fig. 3. Elevated serum $[\mathrm{K}+]$ reduces the $I / R-$ induced increase of the AI.(A) Representative images of TUNEL staining (green) and DAPI (blue) in the cerebral cortices. (B)Values are presented as the mean $\pm \mathrm{SD}$. Elevated serum $[\mathrm{K}+]$ significantly decreased the increase of the AI induced by cerebral I/R. \#\#P<0.01 compared with the Sham group; ${ }^{* *} \mathrm{P}<0.01$ compared with the NS group; $\& \& \mathrm{P}<0.01$ compared with the LD group ( $n=8$ /group).

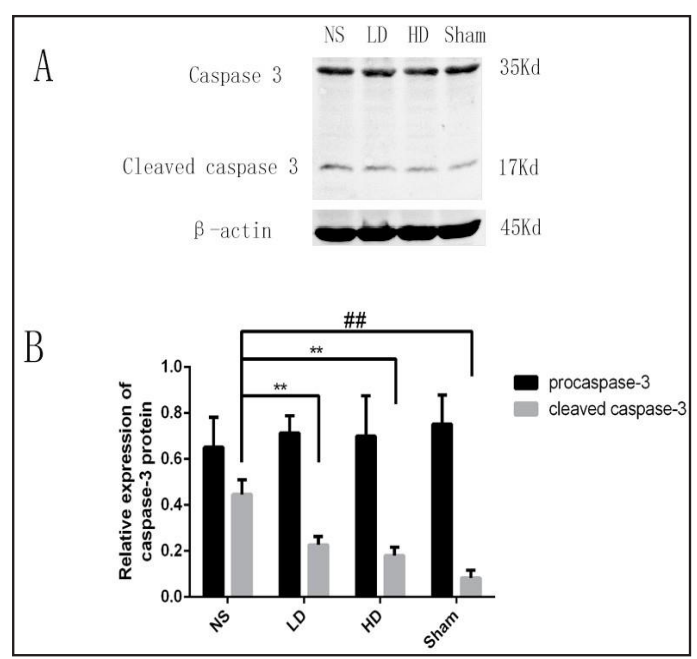

Fig. 4. Elevated serum $[\mathrm{K}+]$ reduces cleavedcaspase-3 expression.(A) Representative western blot bands of procaspase-3 (35 kDa) and cleavedcaspase-3 (17 kDa) in the ischemic cortex at $24 \mathrm{~h}$ after MCAO/R. Corresponding $\beta$-actin (45 kDa) bands were used as loading controls. (B) Relative expression of caspase-3 expression. Data are presented as the mean $\pm \mathrm{SD}$. The gel containing the blot bands of caspase- 3 and cleaved-caspase- 3 was cropped between approximately $10 \mathrm{kDa}$ and 40 $\mathrm{kDa}$ according to the markers, and the other gel containing the blot bands of $\beta$-actin was cropped between approximately $40 \mathrm{kDa}$ to $50 \mathrm{kDa}$ according to the markers.Elevated serum $[\mathrm{K}+]$ significantly reduced the expression of cleaved-caspase-3. \#\#P<0.01 compared with the Sham group; ${ }^{* *} \mathrm{P}<0.01$ compared with the NS group ( $n=8$ /group).

significant difference was observed among the four groups for the expression of caspase-3 (Fig. 4B).

\section{Elevated serum $\left[K^{+}\right]$inhibits the production of ROS}

Basal ROS production of mitochondria was shown by the intensity of red fluorescence in the Sham group (Fig. 5A). The rats in the NS group produced the highest level of ROS, and the production of ROS was reduced in the LD and HD groups compared with the NS group (P $<0.01)$. In addition, the level of ROS was lower in the HD group compared to the LD group $(\mathrm{P}<0.05$, Fig. 5B).

\section{Changes of brain $K^{+}, A T P$, and MDA levels and T-SOD activity}

The changes of brain $\mathrm{K}^{+}$, ATP, and MDA levels and T-SOD activity are presented in Fig. 6AD. At $24 \mathrm{~h}$ of reperfusion, compared with the Sham group, the NS group showed a significant decrease in ATP content and $\mathrm{K}^{+}$level as well as a marked increase in MDA $(\mathrm{P}<0.01)$. Compared with the NS group, both groups with elevated serum $\left[\mathrm{K}^{+}\right]$showed an increase in ATP and $\mathrm{K}^{+}$levels $(\mathrm{P}<0.05$, Fig. 6A, B) as well as reduced MDA level $(\mathrm{P}<0.01$, Fig. $6 \mathrm{C})$, although there was no statistically significant difference between both groups with elevated serum $\left[\mathrm{K}^{+}\right]$. There was no significant difference in T-SOD activity among the NS, LD, and HD groups (Fig. 6D). 
Elevated serum $\left[K^{+}\right]$improves the ultrastructural changes in the mitochondria

The mitochondria from the Sham group displayed normal-appearing membrane integrity, cristae, and homogeneous staining pattern of the matrix (Fig. 7). The mitochondria from the NS group showed severe morphological changes, namely, the mitochondrial membranes were completely collapsed, the cristae were fragmented, and the matrix was electron-lucent (Fig. 7). Elevated serum $\left[\mathrm{K}^{+}\right]$improved the mitochondrial ultrastructural changes in the LD and HD groups. The mitochondria demonstrated moderate swelling with intact mitochondrial membranes, minimally decreased matrix density, and the cristae were nearly intact in most of the mitochondria, especially in the HD group (Fig. 7).

\section{Elevated serum $\left[K^{+}\right]$protects mitochondria by reducing MPTP opening}

Opening of the MPTP was examined by using calcein-AM staining. Mitochondrial fluorescence intensity in the NS group was significantly lower than in the Sham group $(\mathrm{P}<0.01)$, suggesting that $\mathrm{I} / \mathrm{R}$ led to increased MPTP opening, whereas elevated serum $\left[\mathrm{K}^{+}\right]$effectively inhibited I/R-induced MPTP opening when compared to the NS group $(\mathrm{P}<0.01$, Fig. 8$)$. In addition, the intensity of mitochondrial fluorescence was higher in the HD group compared to the LD group $(\mathrm{P}<0.05)$ (Fig. 8).

Elevated serum $\left[\mathrm{K}^{+}\right]$protects
mitochondria by increasing cytochrome $C$ oxidase activity

Mitochondrial complex IV activity was significantly lower in the NS group than in the Sham group $(\mathrm{P}<0.01)$. Elevated serum $\left[\mathrm{K}^{+}\right]$effectively increased mitochondrial complex IV activity when compared with the NS group $(\mathrm{P}<0.01$, Fig. 9). In addition, cytochrome $\mathrm{C}$ oxidase activity was markedly increased in the HD group compared with the LD group $(\mathrm{P}<0.01$, Fig. 9).

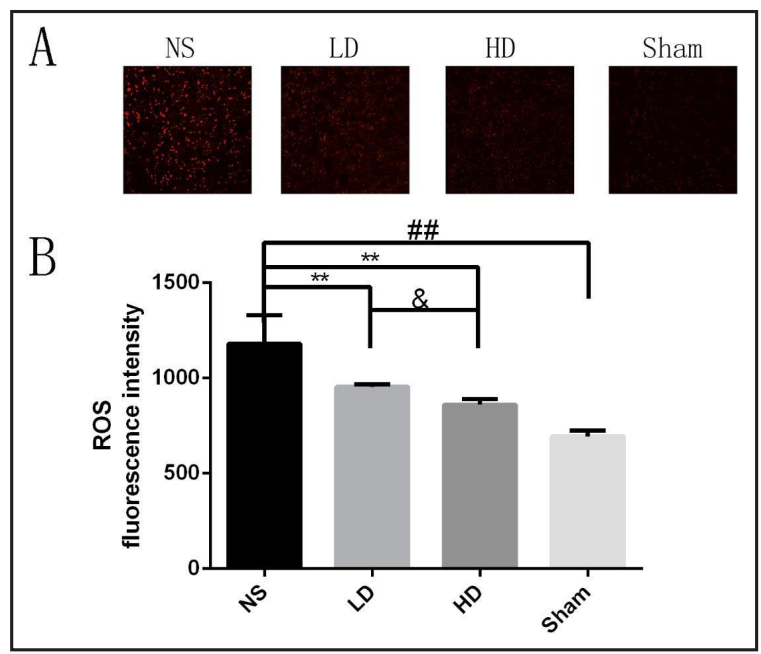

Fig. 5. Elevated serum $[\mathrm{K}+]$ inhibits ROS production. (A) Representative images of DHE staining (red) in the cerebral cortices. (B) Values are presented as the mean \pm SD. \#\#P<0.01 compared with the Sham group; ${ }^{* *} \mathrm{P}<0.01$ compared with the NS group; $\& \mathrm{P}<0.05$ compared with the LD group ( $\mathrm{n}=8$ /group).

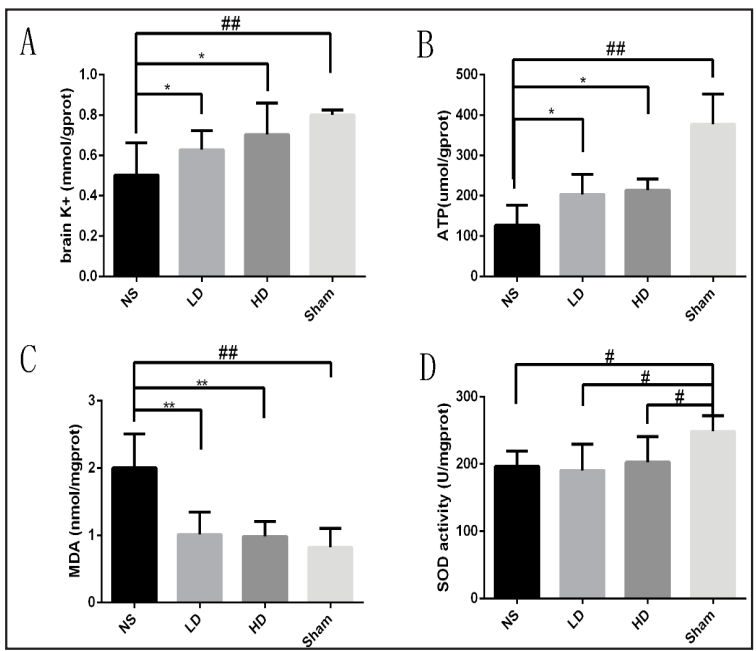

Fig. 6. Changes of brain $\mathrm{K}+$, ATP, and MDA levels and T-SOD activity.Effect of elevated serum [K+] on (A) brain $\mathrm{K}+$ content, (B) ATP level, (C) MDA assay, and (D) T-SOD activity. Values are presented as the mean \pm SD. \#P<0.05 compared with the Sham group; \#\#P<0.01 compared with the Sham group; ${ }^{*} \mathrm{P}<0.05$ compared with the NS group; ${ }^{* *} \mathrm{P}<0.01$ compared with the NS group $(\mathrm{n}=8$ / group). 


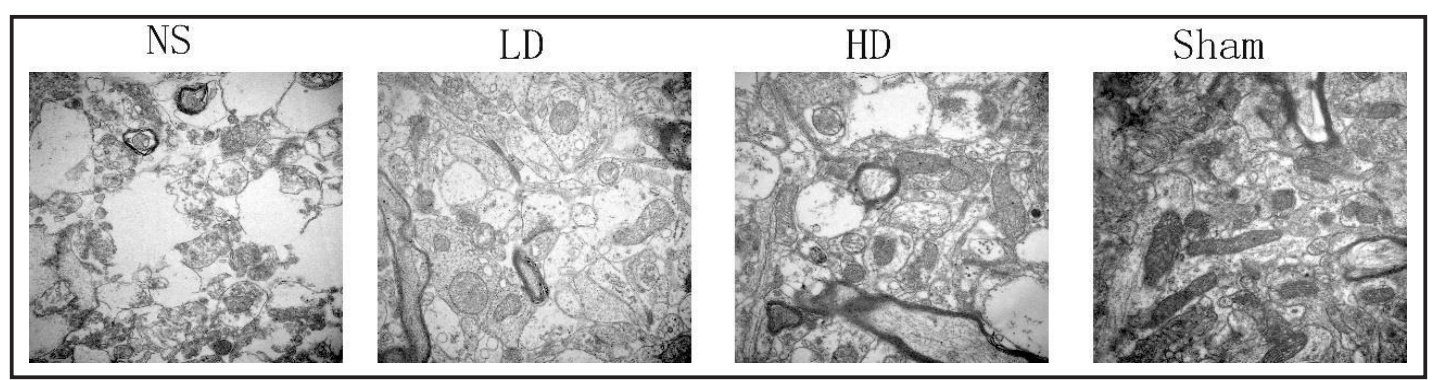

Fig. 7. Elevated serum $[\mathrm{K}+]$ improves the ultrastructural changes in the mitochondria.The mitochondria from the Sham group displayed normal-appearing mitochondrial ultrastructural morphology. The mitochondria from the NS group showed severe morphological changes. Elevated serum [K+] improved the mitochondria ultrastructural changes in the LD and HD groups, especially in the HD group (magnification $\times 30,000)(n=4 /$ group$)$.

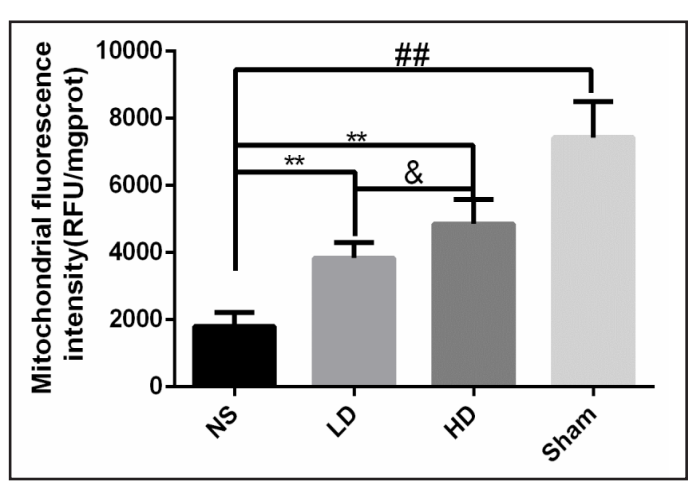

Fig. 8. Elevated serum $[\mathrm{K}+]$ inhibits MPTP opening. Values are presented as the mean $\pm \mathrm{SD}$. \#\#P<0.01 compared with the Sham group; ${ }^{* *} \mathrm{P}<0.01$ compared with the NS group; \&P<0.05 compared with the LD group ( $\mathrm{n}=8$ /group).

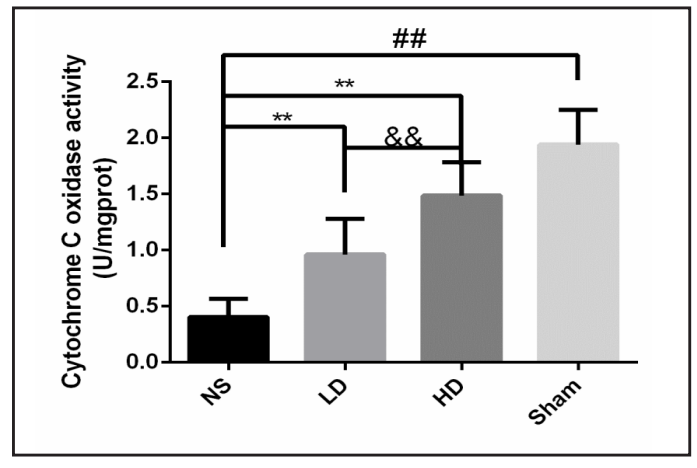

Fig. 9. Elevated serum $[\mathrm{K}+]$ protects mitochondria by increasing cytochrome $\mathrm{C}$ oxidase activity. Values are presented as the mean $\pm \mathrm{SD}$. $\# \# \mathrm{P}<0.01$ compared with the Sham group; ${ }^{* *} \mathrm{P}<0.01$ compared with the NS group; $\& \& \mathrm{P}<0.01$ compared with the LD group ( $n=8 /$ group).

\section{Discussion}

In this study, the HD and LD groups presented with a smaller cerebral infarct volume and reduced AI accompanied with increased cerebral $\left[\mathrm{K}^{+}\right]$when compared with the NS group, suggesting a potential protective effect of elevated serum $\left[\mathrm{K}^{+}\right]$. In addition, the decreased levels of ROS and MPTP opening, increased ATP levels and cytochrome C oxidase activity, and improvement of mitochondrial ultrastructural changes were observed in the HD and LD groups when compared with the NS group, indicating that the protective mechanism may be associated with the preservation of mitochondrial function and structure.

Mitochondria, as a key organelle, participate in cellular metabolism and are the major source of ATP [17]. In the mitochondria, a series of enzyme complexes are known as the electron transport chain (ETC) $[18,19]$. The ETC is responsible for electron transfer via redox reactions. Coupled with the redox reaction, the ETC can also pump protons from the mitochondrial matrix to the intermembrane space between the inner and outer membranes to form a proton gradient. Under normal conditions, a small amount of electrons leak from the ETC to form a low level of ROS, which take part in cellular signaling [20], and the proton gradient can drive ATP synthesis [21]. Dysfunction of the ETC due to ischemia can lead to inefficient electron transfer and the subsequent accumulation of electrons, which could increase the production of ROS dramatically during reperfusion [22, 23]. In addition, a decrease of the proton gradient can hamper ATP synthesis and then disturb normal cellular

\section{KARGER}


metabolism. Accordingly, mitochondrial function is closely related to the ETC.

An in vitro study by Czyz and colleagues showed that higher $\left[\mathrm{K}^{+}\right]$outside mitochondria isolated from liver cells could promote the recovery of the mitochondrial proton gradient [24]. However, its deeper mechanism was not demonstrated fully. For cells in vivo, $\left[\mathrm{K}^{+}\right]$outside the mitochondria represents intracellular $\left[\mathrm{K}^{+}\right]$. On the other hand, cytochrome $\mathrm{C}$ oxidase is recognized as a key enzyme in the ETC. In our present study, cytochrome $\mathrm{C}$ oxidase activity was significantly increased in the presence of higher $\left[\mathrm{K}^{+}\right]$in the HD and LD groups compared with the NS group. Furthermore, higher cytochrome $C$ oxidase activity was coupled with a higher level of ATP and lower level of ROS. We consider that these findings reflected, to some extent, the restoration of cellular metabolism, improvement of electron transfer, and alleviation of oxidative stress.

Theoretically speaking, there is a balance between the generation and elimination of ROS that is maintained by a variety of antioxidant defense mechanisms, one of the most important of which is the SOD family of enzymes. According to the present results, T-SOD activity was significantly decreased in the NS, LD, and HD groups compared to the Sham group, but there was no remarkable difference among the NS, LD, and HD groups. On the basis of the study by Czyz et al. [24]. and our present findings, we can further deduce that the restoration of intracellular potassium (or $\left[\mathrm{K}^{+}\right]$outside the mitochondria]) by elevated serum $\left[\mathrm{K}^{+}\right]$can promote the recovery of mitochondrial function by improving the activity of ETC enzymes rather than via the ROS scavenging mechanism by SOD. Therefore, the alleviation of cerebral damage caused by oxidative stress was eventually realized.

The homeostasis of intracellular potassium plays an important role in cell survival and apoptosis [24]. Previous in vitro studies showed that intracellular potassium efflux occurred during the early stage of cellular apoptosis, and decreased $\left[\mathrm{K}^{+}\right]$could facilitate the activation of apoptosis-related enzymes, such as caspase-3 [25, 26]. Nevertheless, both the inhibition of intracellular potassium efflux and the restoration of $\left[\mathrm{K}^{+}\right]$could alleviate apoptosis $[27$, 28]. Similarly, an in vivo CA model induced by excessive serum [ $\left.\mathrm{K}^{+}\right]$exhibited the favorable restoration of spontaneous circulation and reduced cerebral damage post-CA compared with a CA model induced by electric stimulation of the cardiac endocardium. We speculated that the difference in serum $\left[\mathrm{K}^{+}\right]$between both CA models may have a key role in the protective mechanism.

The MPTP is localized on the mitochondrial membrane and is kept closed in normal conditions. When oxidative stress occurs, a large number of ROS are formed that then damage mitochondrial membrane structure [29] and increase MPTP opening [30], resulting in the loss of membrane potential and the increased production of free radicals $[21,22]$. Increased mitochondrial permeability also increases the release of pro-apoptotic factors', such as cytochrome $\mathrm{C}$, into the cytoplasm, which can activate the apoptosis-related enzyme caspase-3 [31]. Similarly, we restored $\mathrm{K}^{+}$homeostasis in neurons by elevating serum $\left[\mathrm{K}^{+}\right]$, which promotes the recovery of mitochondrial function and subsequently reduces ROS formation. Thus, MPTP opening was decreased and mitochondrial structure was improved, which were followed by the decreased expression of a pro-apoptotic enzyme.

In our preliminary experiment, the venous administration of a $2.5 \% \mathrm{KCl}(80 \mathrm{mg} / \mathrm{kg})$ solution at a rate of $8 \mathrm{~mL} / \mathrm{h}$ did not affect hemodynamic stability, while a higher dosage of $\mathrm{KCl}(120 \mathrm{mg} / \mathrm{kg})$ caused heart arrhythmia and hemodynamic instability. Therefore, KCl solutions at a concentration $>80 \mathrm{mg} / \mathrm{kg}$ were excluded from the present study, although they seemed to present better protection to rats subjected to cerebral IRI. Nevertheless, in our present study, serum $\left[\mathrm{K}^{+}\right]$was less than $5.5 \mathrm{mmol} / \mathrm{L}$ in rats treated with $\mathrm{KCL}$, which did not reach the level required for hyperkalemia. We hypothesize that rats may benefit from a serum $\left[\mathrm{K}^{+}\right]$higher than the normal range. In addition, it must be noted that KCL application was only a means to regulate serum $\left[\mathrm{K}^{+}\right]$in our present study, and more effective and safer medicines to achieve the same aim could be a potential option.

The novelties of our present work are as follow. First, we have demonstrated that elevated serum $\left[\mathrm{K}^{+}\right]$could exert an effect on potassium homeostasis in neurons and had a neuroprotective effect in vitro based on previous in vivo studies. In addition, we provided 


\section{Cellular Physiology Cell Physiol Biochem 2018;48:1664-1674

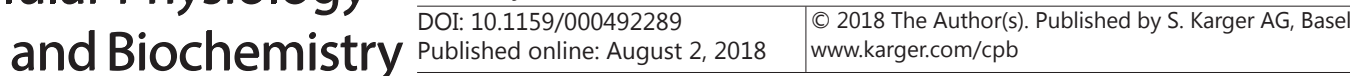

Li et al.: Cerebral Protection by Serum Potassium Regulation

evidence showing that the restoration of enzymatic activity in the ETC may be the underlying mechanism by which elevated serum $\left[\mathrm{K}^{+}\right]$alleviated cerebral IRI. Further studies are needed to answer the question how elevated serum $\left[\mathrm{K}^{+}\right]$restores intracellular $\mathrm{K}+$ homeostasis.

\section{Conclusion}

Our findings suggested that the elevation of serum potassium by the venous administration of a $\mathrm{KCl}$ solution at the moment of reperfusion after 90 min MCAO can alleviate cerebral apoptosis. The neuroprotective mechanism may be associated with the decreased loss of intracellular potassium and the preservation of mitochondrial function and structure.

\section{Acknowledgements}

This research was supported by the National Natural Science Foundation of China (81360286) and Guangxi Natural Science Foundation (2016GXNSFAA380285). We thank Dr. Bernard Albert Goodman for his assistance with editing this paper. Nuo Li and Sina Qin contributed equally to this paper. Nuo Li and Sina Qin contributed equally to writing the manuscript. Nuo Li, Sina Qin, Lu Xie, Tao Qin, Yegui Yang, and Wei Fang all performed the experiments. Sina Qin prepared the figures. Menghua Chen and Nuo Li designed the study.

\section{Disclosure Statement}

The authors have no conflicts of interest to declare.

\section{References}

1 Lust WD, Taylor C, Pundik S, Selman WR, Ratcheson RA: Ischemic cell death: dynamics of delayed secondary energy failure during reperfusion following focal ischemia. Metab Brain Dis 2002;17:113-121.

2 Schaller B, Graf R: Cerebral ischemia and reperfusion: the pathophysiologic concept as a basis for clinical therapy. J Cereb Blood Flow Metab 2004;24:351-371.

3 Oliver CN, Starke-Reed PE, Stadtman ER, Liu GJ, Carney JM, Floyd RA: Oxidative damage to brain proteins, loss of glutamine synthetase activity, and production of free radicals during ischemia/reperfusion-induced injury to gerbil brain. Proc Natl Acad Sci U S A 1990;87:5144-5147.

4 Li J, Ma X, Yu W, Lou Z, Mu D, Wang Y, Shen B, Qi S: Reperfusion promotes mitochondrial dysfunction following focal cerebral ischemia in rats. PloS one 2012;7:e46498.

5 Turrens JF: Mitochondrial formation of reactive oxygen species. J Physiol 2003;552:335-344.

6 Sanderson TH, Reynolds CA, Kumar R, Przyklenk K, Huttemann M: Molecular mechanisms of ischemiareperfusion injury in brain: pivotal role of the mitochondrial membrane potential in reactive oxygen species generation. Mol Neurobiol 2013;47:9-23.

7 Chen H, Yoshioka H, Kim GS, Jung JE, Okami N, Sakata H, Maier CM, Narasimhan P, Goeders CE, Chan PH: Oxidative stress in ischemic brain damage: mechanisms of cell death and potential molecular targets for neuroprotection. Antioxid Redox Signal 2011;14:1505-1517.

8 Yu SP, Canzoniero LM, Choi DW: Ion homeostasis and apoptosis.Curr Opin Cell Biol 2001;13:405-411.

-9 Yu SP: Regulation and critical role of potassium homeostasis in apoptosis. Prog Neurobiol 2003;70:363386.

10 Yu SP, Yeh CH, Sensi SL, Gwag BJ, Canzoniero LM, Farhangrazi ZS, Ying HS, Tian M, Dugan LL, Choi DW: Mediation of neuronal apoptosis by enhancement of outward potassium current. Science 1997;278:114117. 


\section{Cellular Physiology Cell Physiol Biochem 2018;48:1664-1674 \begin{tabular}{ll|l} 
DOI: 10.1159/000492289 2018 & $\begin{array}{l}\text { O } 2018 \text { The Author(s). Published by S. Karger AG, Basel } \\
\text { www.karger.com/cpb }\end{array}$ \\
and Biochemistry Published online: August 2, 2018
\end{tabular}}

Li et al.: Cerebral Protection by Serum Potassium Regulation

11 Karki P, Seong C, Kim JE, Hur K, Shin SY, Lee JS, Cho B, Park IS: Intracellular K(+) inhibits apoptosis by suppressing the Apaf-1 apoptosome formation and subsequent downstream pathways but not cytochrome c release. Cell Death Differ 2007;14:2068-2075.

12 Song L, Weil MH, Tang W, Sun S, Pellis T: Cardiopulmonary resuscitation in the mouse. J Appl Physiol 2002;93:1222-1226.

13 Angelos MG, Butke RL, Panchal AR, Torres CA, Blumberg A, Schneider JE, Aune SE: Cardiovascular response to epinephrine varies with increasing duration of cardiac arrest. Resuscitation 2008;77:101-110.

14 Kofler J, Hattori K, Sawada M, DeVries AC, Martin LJ, Hurn PD, Traystman RJ: Histopathological and behavioral characterization of a novel model of cardiac arrest and cardiopulmonary resuscitation in mice. J Neurosci Methods 2004;136:33-44.

15 Longa EZ, Weinstein PR, Carlson S, Cummins R: Reversible middle cerebral artery occlusion without craniectomy in rats. Stroke 1989;20:84-91.

16 Xing B, Chen H, Zhang M, Zhao D, Jiang R, Liu X, Zhang S: Ischemic postconditioning inhibits apoptosis after focal cerebral ischemia/reperfusion injury in the rat. Stroke 2008;39:2362-2369.

17 Nath S, Villadsen J: Oxidative phosphorylation revisited. Biotechnol Bioeng 2015;112:429-437.

18 Diaz F, Enriquez JA, Moraes CT: Cells lacking Rieske iron-sulfur protein have a reactive oxygen speciesassociated decrease in respiratory complexes I and IV. Mol Cell Biol 2012;32:415-429.

19 Lobo-Jarne T, Ugalde C: Respiratory chain supercomplexes: Structures, function and biogenesis. Semin Cell Dev Biol 2018;76:179-190.

20 Circu ML, Aw TY: Reactive oxygen species, cellular redox systems, and apoptosis. Free Radic Biol Med 2010;48:749-762.

-21 Turrens JF: Superoxide production by the mitochondrial respiratory chain. Biosci Rep 1997;17:3-8.

22 Piantadosi CA, Zhang J: Mitochondrial generation of reactive oxygen species after brain ischemia in the rat. Stroke 1996;27:327-331; discussion 332.

23 Nohl H, Gille L, Staniek K: The mystery of reactive oxygen species derived from cell respiration. Acta Biochim Pol 2004;51:223-229.

24 Czyz A, Szewczyk A, Nalecz MJ, Wojtczak L: The role of mitochondrial potassium fluxes in controlling the protonmotive force in energized mitochondria. Biochem Biophys Res Commun 1995;210:98-104.

25 Barbiero G, Duranti F, Bonelli G, Amenta JS, Baccino FM: Intracellular ionic variations in the apoptotic death of L cells by inhibitors of cell cycle progression. Exp Cell Res 1995;217:410-418.

-26 Benson RS, Heer S, Dive C, Watson AJ: Characterization of cell volume loss in CEM-C7A cells during dexamethasone-induced apoptosis. Am J Physiol 1996;270:C1190-1203.

27 Nietsch HH, Roe MW, Fiekers JF, Moore AL, Lidofsky SD: Activation of potassium and chloride channels by tumor necrosis factor alpha. Role in liver cell death. J Biol Chem 2000;275:20556-20561.

28 Wei L, Yu SP, Gottron F, Snider BJ, Zipfel GJ, Choi DW: Potassium channel blockers attenuate hypoxia- and ischemia-induced neuronal death in vitro and in vivo. Stroke 2003;34:1281-1286.

29 Chen Q Gong B, Almasan A: Distinct stages of cytochrome c release from mitochondria: evidence for a feedback amplification loop linking caspase activation to mitochondrial dysfunction in genotoxic stress induced apoptosis. Cell Death Differ 2000;7:227-233.

30 Zhou H, Hu S, Jin Q, Shi C, Zhang Y, Zhu P, Ma Q, Tian F, Chen Y: Mff-Dependent Mitochondrial Fission Contributes to the Pathogenesis of Cardiac Microvasculature Ischemia/Reperfusion Injury via Induction of mROS-Mediated Cardiolipin Oxidation and HK2/VDAC1 Disassociation-Involved mPTP Opening. J Am Heart Assoc 2017;6

-31 Ott M, Gogvadze V, Orrenius S, Zhivotovsky B: Mitochondria, oxidative stress and cell death. Apoptosis 2007;12:913-922. 